\title{
MULTILINEAR MAPPINGS OF NUCLEAR AND INTEGRAL TYPE
}

\author{
RAYMUNDO ALENCAR ${ }^{1}$
}

\begin{abstract}
We define vector-valued multilinear mappings of nuclear and integral type and establish conditions for its coinciding spaces.
\end{abstract}

Introduction. If $E$ and $F$ are Banach spaces then $L_{N}(E, F), L_{P I}(E, F)$, and $L_{G I}(E, F)$ denote the Banach spaces of all nuclear, Pietsch-integral, and Grothendieck-integral linear mappings from $E$ into $F$ endowed with their respective norms. We always have the continuous inclusions $L_{N}(E, F) \subset L_{P I}(E, F) \subset$ $L_{G I}(E, F)$, and we are interested in finding conditions on $E$ or $F$ which guarantee the opposite inclusions. Let us recall the following results from the book of Diestel and Uhl [3].

Theorem A [3, Theorem VI. 4.8]. A Banach space $F$ has the Radon-Nikodym property if and only if for every Banach space $E$, each $T \in L_{P I}(E, F)$ is nuclear. In this case the identity mapping, $L_{N}(E, F) \rightarrow L_{P I}(E, F)$ is an isometry.

Theorem B [3, Theorem VIII. 4.6]. Let E be a Banach space whose dual $E^{*}$ has the approximation property. Then $E^{*}$ has the Radon-Nikodym property if and only if for every Banach space $F$, each $T \in L_{G I}(E, F)$ is nuclear. In this case the identity mapping $L_{N}(E, F) \rightarrow L_{G I}(E, F)$ is an isometry.

Since the identity $L_{P I}(E, F)=L_{G I}(E, F)$ holds, e.g. whenever $F$ is a dual space, Theorem B may be regarded as a sort of dual of Theorem A. In this paper we establish the following variant of Theorem B, which does not involve the approximation property.

THEOREM 1.3. The dual $E^{*}$ of a Banach space $E$ has the Radon-Nikodym property if and only if, for every Banach space $F$, each $T \in L_{P I}(E, F)$ is nuclear. In this case the identity mapping $L_{N}(E, F) \rightarrow L_{P I}(E, F)$ is an isometry.

Curiously enough, Theorem A does not extend to the case of bilinear mappings, as we shall see in Remark 2.4; but with the obvious notation, Theorem 1.3 can be extended to the case of bilinear mappings as follows:

Received by the editors March 25, 1984.

1980 Mathematics Subject Classification. Primary 15A69; Secondary 46B22, 46G10.

${ }^{1}$ Research supported in part by FAPESP (Brazil) when the author was visiting Kent State University. Permanent address: Universidade de Sāo Paulo, Instituto de Matematica e Estatistica, Caixa Postal 20570, 05508-Sāo Paulo, Brasil 
THEOREM 2.3. Let $E$ and $F$ be Banach spaces whose duals $E^{*}$ and $F^{*}$ have the Radon-Nikodym property. Then, for every Banach space $G$, each $T \in L_{P I}(E, F ; G)$ is nuclear. In this case the identity mapping $L_{N}(E, F ; G) \rightarrow L_{P I}(E, F ; G)$ is an isometry.

The key to the proofs of Theorems 1.3 and 2.3 is a result of Edgar [5]. Alternatively, we could have used a closely related result of Schwartz [8].

1. The linear case. The letters $E, F$, and $G$ will always denote Banach spaces. For the terminology of vector measures, Pietsch-integral linear mappings, and Grothendieck-integral linear mappings, we refer the reader to the book of Diestel and Uhl [3].

1.1. Proposition. If $T \in L(E, F)$ is Pietsch-integral, then its adjoint $T^{*} \in$ $L\left(F^{*}, E^{*}\right)$ is also Pietsch-integral.

Proof. By [3, Theorem VI. 3.10] a mapping $T \in L(E, F)$ is Pietsch-integral if and only if $T$ admits a factorization of the form

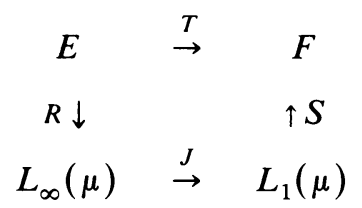

where $\mu$ is a regular, nonnegative Borel measure on some compact Hausdorff space $\Omega, R$ and $S$ are continuous linear mappings, and $J$ is the natural inclusion.

Therefore it suffices to take adjoints in the above diagram and apply Lemma 1.2 below with $X=L_{1}(\mu), Y=L_{\infty}(\mu), J: Y \rightarrow X$ the natural inclusion, and $I: X^{*} \rightarrow Y$ the natural isomorphism.

1.2. Lemma. Let $J \in L(Y, X)$, where $X$ and $Y$ are Banach spaces. If there exists $I \in L\left(X^{*}, Y\right)$ such that $\varphi(J I \psi)=\psi(J I \varphi)$ for all $\varphi, \psi \in X^{*}$, then the following diagram is commutative:

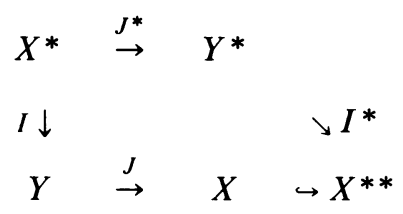

Proof. For $\varphi, \psi \in X^{*}$, we have

$$
\left(I^{*} J^{*} \varphi\right)(\psi)=J{ }^{*}(I \psi)=\varphi(J I \psi)=\psi(J I \varphi) .
$$

1.3. TheOREM. The dual $E^{*}$ of a Banach space $E$ has the Radon-Nikodym property if and only if, for every Banach space $F$, each $T \in L_{P I}(E, F)$ is nuclear. In this case the identity mapping $L_{N}(E, F) \rightarrow L_{P I}(E, F)$ is an isometry. 
Proof. First assume $E^{*}$ has the Radon-Nikodym property. If $T \in L_{P I}(E, F)$ then there exists a regular countably additive $F$-valued Borel measure of bounded variation $\nu$ on $U_{E^{*}}$ (the closed unit ball of $E^{*}$ with the weak*-topology) such that

$$
T(X)=\int_{U_{E^{*}}} \varphi(x) d \nu(\varphi) \text { for all } x \in E,
$$

then the variation $|\nu|$ is a regular positive Borel measure on $U_{E^{*}}$. Since $E^{*}$ has the Radon-Nikodym property, a result of Edgar [5, Theorem 1.5] implies at once that the inclusion mapping

$$
f=\left(U_{E^{*}}, \text { weak }^{*}\right) \rightarrow\left(E^{*}, \text { norm }\right)
$$

is universally measurable-in particular, $|\nu|$-measurable.

Since $\|f(\varphi)\|=\|\varphi\| \leqslant 1$ on $U_{E^{*}}$, it is clear that $f$ is Bochner integrable with respect to $|\nu|$.

Let $\varepsilon>0$ be given. By [3, Lemma VI. 4.3] there exist a sequence $\left(\varphi_{n}\right)$ in $E^{*}$ and a sequence of Borel sets $A_{n} \subset U_{E^{*}}$ such that

(i) the series $\sum_{n=1}^{\infty} \varphi_{n} x_{A_{n}}$ converges absolutely to $f|\nu|$-a.e.,

(ii) $\sum_{n=1}^{\infty}\left\|\varphi_{n}\right\||\nu|\left(A_{n}\right) \leqslant|\nu|\left(U_{E^{*}}\right)+\varepsilon$

(iii) $\sum_{n=1}^{\infty}\left\|\varphi_{n}\right\| x_{A_{n}}(\varphi) \leqslant 1+\varepsilon /\left(|\nu|\left(U_{E^{*}}\right)\right)|\nu|$-a.e.

By (i), for each $x \in E$ we can write

$$
T x=\int_{U_{E^{*}}} \sum_{n=1}^{\infty} \varphi_{n}(x) x_{A_{n}}(\varphi) d \nu(\varphi) .
$$

If we define $f_{k}=\sum_{n=1}^{k} \varphi_{n} x_{A_{n}}$ for $k=1,2, \ldots$, then, by (iii), the sequence $\left(f_{k}\right)$ is uniformly bounded $|\nu|$-a.e. We may then apply Bartle's bounded convergence Theorem [3, Theorem II. 4.1] to obtain, for every $x \in E$,

$$
\begin{aligned}
T x & =\sum_{n=1}^{\infty} \varphi_{n}(x) \int_{U_{E^{*}}} x_{A_{n}}(\varphi) d \nu(\varphi) \\
& =\sum_{n=1}^{\infty} \varphi_{n}(x) \nu\left(A_{n}\right) .
\end{aligned}
$$

From (ii) we see that

$$
\sum_{n=1}^{\infty}\left\|\varphi_{n}\right\|\left\|\nu\left(A_{n}\right)\right\| \leqslant|\nu|\left(U_{E^{*}}\right)+\varepsilon
$$

and, hence, $T \in L_{N}(E, F)$ and $\|T\|_{N} \leqslant\|T\|_{P I}$, as asserted.

Conversely, assume that for every Banach space $F$, each $T \in L_{P I}(E, F)$ is nuclear. In view of Theorem $\mathrm{A}$, to prove that $E^{*}$ has the Radon-Nikodym property, it is sufficient to show that each $T \in L_{P I}\left(F, E^{*}\right)$ is nuclear.

Let $T \in L_{P I}\left(F, E^{*}\right)$. By Proposition $1.1, T^{*} \in L_{P I}\left(E^{* *}, F^{*}\right)$. If we set $S=$ $\left.T^{*}\right|_{E}$, then clearly $S \in L_{P I}\left(E, F^{*}\right)$ and, hence, $S \in L_{N}\left(E, F^{*}\right)$ by the converse hypothesis. Hence, $S^{*} \in L_{N}\left(F^{* *}, E^{*}\right)$ by [7, Proposition 3.1.8].

Since we can readily see that $\left.S^{*}\right|_{F}=T$, we conclude that $T \in L_{N}\left(F, E^{*}\right)$, and the proof is complete.

1.4. RemarK. [3, Corollary VIII. 2.11] asserts that a mapping $T \in L(E, F)$ is Grothendieck-integral if and only if its adjoint $T^{*}$ is Grothendieck-integral, but the 
corresponding result for Pietsch-integral mappings is not true in general. Indeed, Figiel and Johnson [6] have found a Banach space $E$ with a separable dual and a nonnuclear mapping $T \in L(E, F)$ whose adjoint $T^{*}$ is nuclear. Thus, in particular, $T^{*}$ is Pietsch-integral and Grothendieck-integral. Since $E^{*}$ has the Radon-Nikodym property, $T$ cannot be Pietsch-integral, for otherwise $T$ would be nuclear, by Theorem 1.3. We see also that $T$ is an example of a Grothendieck-integral mapping which is not Pietsch-integral.

2. The bilinear case. Let $L(E, F ; G)$ denote the space of all continuous bilinear mappings $T: E \times F \rightarrow G$. We recall, see [2, Definition 1.26], that a mapping $T \in L(E, F ; G)$ is nuclear if there are sequences $\left(\varphi_{n}\right)$ in $E^{*},\left(\psi_{n}\right)$ in $F^{*}$, and $\left(c_{n}\right)$ in $G$ with

$$
\sum_{n=1}^{\infty}\left\|\varphi_{n}\right\|\left\|\psi_{n}\right\|\left\|c_{n}\right\|<\infty
$$

such that

$$
T(x, y)=\sum_{n=1}^{\infty} \varphi_{n}(x) \psi_{n}(y) c_{n} \text { for all }(x, y) \in E \times F .
$$

Let $L_{N}(E, F ; G)$ denote the space of all nuclear bilinear mappings $T: E \times F \rightarrow G$ endowed with the norm

$$
\|T\|_{N}=\inf \sum_{n=1}^{\infty}\left\|\varphi_{n}\right\|\left\|\psi_{n}\right\|\left\|c_{n}\right\|,
$$

where the infimum is taken over all sequences $\left(\varphi_{n}\right),\left(\psi_{n}\right)$, and $\left(c_{n}\right)$ which satisfy the definition.

2.1. Definition. A mapping $T \in L(E, F ; G)$ is said to be Pietsch-integral if there exists a regular countably additive, $G$-valued Borel measure $\nu$ of bounded variation on the product $U_{E^{*}} \times U_{F^{*}}$ such that

$$
T(x, y)=\int_{U_{E^{*}} \times U_{F^{*}}} \varphi(x) \psi(y) d \nu(\varphi, \psi) \quad \text { for all }(x, y) \in E \times F .
$$

Let $L_{P I}(E, F ; G)$ denote the space of all Pietsch-integral bilinear mappings $T$ : $E \times F \rightarrow G$ endowed with the norm $\|T\|_{P I}=\inf |\nu|\left(U_{E^{*}} \times U_{F^{*}}\right)$, where the infimum is taken over all vector measures $\nu$ satisfying the definition.

2.2. RemarK. If $G=\mathbf{K}$ is the scalar field, then $L_{P I}(E, F ; \mathbf{K})$ coincides with the space of all bilinear forms on $E \times F$ which are integral in the sense of Grothendieck (see [3, Theorem VIII. 2.5 and Definition VIII. 2.6]).

We always have the inclusion $L_{N}(E, F ; G) \subset L_{P I}(E, F ; G)$ and $\|T\|_{P I} \leqslant\|T\|_{N}$ for all $T \in L_{N}(E, F ; G)$.

Our next result extends Theorem 1.3 to the case of bilinear mappings.

2.3. Theorem. Let $E$ and $F$ be Banach spaces whose duals $E^{*}$ and $F^{*}$ have the Radon-Nikodym property. Then for every Banach space $G$, each $T \in L_{P I}(E, F ; G)$ is nuclear. In this case the identity mapping $L_{N}(E, F ; G) \rightarrow L_{P I}(E, F ; G)$ is an isometry. 
Proof. If $T \in L_{P I}(E, F ; G)$ then there is a regular, countably additive, $G$-valued Borel measure $\nu$ of bounded variation on the product $U_{E^{*}} \times U_{F^{*}}$ such that

$$
T(x, y)=\int_{U_{E^{*}} \times U_{F^{*}}} \varphi(x) \psi(y) d \nu(\varphi, \psi) \quad \text { for all }(x, y) \in E \times F .
$$

As we saw in the proof of Theorem 1.3, the inclusion mappings $f: U_{E^{*}} \rightarrow E^{*}$ and $g: U_{F^{*}} \rightarrow F^{*}$ are both universally measurable.

We define a regular positive Borel measure $\lambda$ on $U_{E^{*}}$ and a regular positive Borel measure $\mu$ on $U_{F^{*}}$ by

$$
\begin{array}{ll}
\lambda(A)=|\nu|\left(A \times U_{F^{*}}\right) & \text { for each Borel set } A \subset U_{E^{*}}, \\
\mu(B)=|\nu|\left(U_{E^{*}} \times B\right) & \text { for each Borel set } B \subset U_{F^{*}} .
\end{array}
$$

Thus, $f$ is $\lambda$-measurable and $g$ is $\mu$-measurable, and one can readily verify that the tensor product mapping

$$
f \otimes g: U_{E^{*}} \times U_{F^{*}} \rightarrow E^{*} \hat{\otimes}_{\pi} F^{*}
$$

is $|\nu|$-measurable.

Since $\|f(\varphi) \otimes g(\psi)\|=\|\varphi \otimes \psi\| \leqslant 1$ on $U_{E^{*}} \times U_{F^{*}}$, we see at once that $f \otimes g$ is Bochner integrable with respect to $|\nu|$. Thus, we may apply [3, Lemma VI. 4.3] to the mapping $f \otimes g$, and an argument similar to the one in the proof of Theorem 1.3 shows that, given $\varepsilon>0$, we can find a sequence $\left(u_{n}\right)$ in $E^{*} \hat{\otimes}_{\pi} F^{*}$ and a sequence of Borel sets $A_{n} \subset U_{E^{*}} \times U_{F^{*}}$ with

$$
\sum_{n=1}^{\infty}\left\|u_{n}\right\||\nu|\left(A_{n}\right) \leqslant|\nu|\left(U_{E^{*}} \times U_{F^{*}}\right)+\varepsilon
$$

such that

$$
T(x, y)=\sum_{n=1}^{\infty} u_{n}(x, y) \nu\left(A_{n}\right) \quad \text { for all }(x, y) \in E \times F
$$

By [7, Theorem 7.5.1] each $u_{n}$ can be represented in the form

$$
u_{n}=\sum_{k=1}^{\infty} \varphi_{n k} \otimes \psi_{n k}
$$

where $\varphi_{n k} \in E^{*}$ and $\psi_{n k} \in F^{*}$ verify the conditions

$$
\sum_{k=1}^{\infty}\left\|\varphi_{n k}\right\|\left\|\psi_{n k}\right\| \leqslant\left\|u_{n}\right\|+\varepsilon \cdot 2^{-n} \quad \text { for every } n=1,2, \ldots .
$$

Thus, for all $(x, y) \in E \times F$ we can write

$$
T(x, y)=\sum_{n=1}^{\infty} \sum_{k=1}^{\infty} \varphi_{n k}(x) \psi_{n k}(y) \nu\left(A_{n}\right)
$$

and

$$
\sum_{n=1}^{\infty} \sum_{k-1}^{\infty}\left\|\varphi_{n k}\right\|\left\|\psi_{n k}\right\||\nu|\left(A_{n}\right) \leqslant(1+\varepsilon)|\nu|\left(U_{E^{*}} \times U_{F^{*}}\right) .
$$

The proof is complete. 
2.4. REMARK. Theorem 2.3 may be regarded as an extension of Theorem 1.3 to the case of bilinear mappings. On the other hand, Theorem A does not extend to the case of bilinear mappings in general, i.e., the Radon-Nikodym property for $G$ alone does not imply the equality $L_{N}(E, F ; G)=L_{P I}(E, F ; G)$.

Indeed, let $E=F=C[0,1]$ be the space of all complex-valued continuous functions on [0,1] and let $G=\mathrm{C}$. If we define $T(f, g)=\int_{0}^{1} f(t) g(t) d t$, then one can see [Köthe, Vol. II, p. 307] that $T$ is a Pietsch-integral bilinear form on $E \times E$, but $T$ is not nuclear, and this is sufficient to check that the linear mapping $\tilde{T}: E \rightarrow E^{*}$, defined by $\tilde{T}(f)(g)=T(f, g), f, g \in E$, is not compact (details in [1]).

Finally, we remark that the results in this section extend in an obvious way to the case of multilinear mappings.

ACKnowledgement. The author thanks Professor Jorge Mujica for introducing him to this subject.

\section{REFERENCES}

1. R. Alencar, Aplicaçoes nucleares e integrais e a propiedade de Radon Nikodym, Thesis, Univ. de Såo Paulo, 1982.

2. S. Dineen, Complex analysis in locally convex spaces, North-Holland, Amsterdam, 1981.

3. J. Diestel and J. Uhl, Vector measures, Math. Surveys, Amer. Math. Soc., vol. 15, Providence, R.I., 1977.

4. N. Dunford and J. T. Schwartz, Linear operators. I, Interscience, New York, 1957.

5. G. Edgar, Measurability in a Banach space, Indiana Univ. Math. J. 26 (1977), 663-677.

6. T. Figel and W. Johnson, The approximation property does not imply the bounded approximation property, Proc. Amer. Math Soc. 41 (1973), 197-200.

7. A. Pietsch, Nuclear locally convex spaces, Springer, Berlin, 1972.

8. L. Schwartz, Proprieté de Radon Nikodym, Sėminaire Maurey-Schwartz 1974-1975, Exposé Nº . V-VI.

Department of Mathematical Sciences, Kent State University, Kent, Ohio 44242 